\title{
Uncovering the genetic background of natural anticoagulant deficiencies: time to look behind the scenes
}

\author{
Zsuzsa Bagoly \\ Division of Clinical Laboratory Sciences, University of Debrecen, Faculty of Medicine, Debrecen, Hungary
}

Correspondence to:

Zsuzsa Bagoly, MD, PhD,

University of Debrecen, Faculty of Medicine, Division of Clinical Laboratory Sciences, 98 Nagyerdei krt., 4032 Debrecen, Hungary, phone: +36 52431956, e-mail: bagoly@med.unideb.hu Received: July 27, 2017. Accepted: July 27, 2017. Published online: August 3, 2017. Conflict of interest: none declared. Pol Arch Intern Med. 2017; 127 (7-8): 465-467 doi:10.20452/pamw.4069 Copyright by Medycyna Praktyczna, Kraków 2017
Understanding the causes of excessive blood clotting has been a long-time challenge. As early as in 1856, Rudolf Virchow postulated in his well known theory of the triad that one of the key components in the etiology of thrombosis is the "change in the composition of blood". ${ }^{1} \mathrm{Nev}$ ertheless, more than 100 years had to pass after this groundbreaking observation for the first case of inherited antithrombin (AT) deficiency to be published by Egeberg in 1965 . $^{2}$ The first functional AT defect, named AT Budapest was published by Sas et $\mathrm{al}^{3}$ in 1974, followed by a series of reports on protein C (PC) and protein S (PS) deficiency in the 1980s. ${ }^{1,4,5}$ Today, the term "thrombophilia" is used to describe a tendency to develop venous thromboembolism due to abnormalities of blood coagulation that can be inherited, acquired, or both. ${ }^{6}$ Inherited thrombophilias include loss-of-function mutations of the genes encoding the natural anticoagulant proteins leading to AT, PC, and PS deficiencies, as well as the gain-of-function mutations comprising of the relatively frequent factor $V$ Leiden mutation and a mutation in the prothrombin gene (FII20210A). In addition, data are accumulating on further hereditary factors, including the non-O blood types. ${ }^{1,6,7}$

Our knowledge on inherited deficiencies of natural anticoagulants has evolved greatly since the first publication by Egeberg. ${ }^{2}$ However, due to the very low prevalence of these disorders in the general population, the majority of this knowledge is still based on case reports and expert opinions. ${ }^{7.8}$ High-quality research on the genotype-phenotype associations and structural-functional studies are of great importance when attempting to unravel the pathophysiology of these rare diseases. Surprisingly, until now only a few case reports have been published on Polish AT/PC/PS-deficient patients with known causal mutations. ${ }^{9-11}$ In this issue of the Polish Archives of Internal Medicine (Pol Arch Intern Med), Wypasek et $\mathrm{al}^{12}$ published the first large and comprehensive study analyzing the genetic background of natural anticoagulant deficiencies in the Polish Slavic population. The authors evaluated the causal genetic background of 90 unrelated patients (mean [SD] age, 40.1 [13.2] years) with AT $(n=35), P C$ ( $\mathrm{n}=28$ ), or PS ( $\mathrm{n}=27$ ) deficiencies, screening for mutations using the Sanger sequencing and multiplex ligation-dependent probe amplification. Twenty novel mutations were described, all present in a heterozygous form. The frequency of missense, nonsense, and splice-site mutations was similar for all 3 genes/proteins.

Currently, more than 250 loss-of-function mutations have been identified in the AT gene (SERPIN1), located at chromosome 1q 23-25. ${ }^{6}$ Among the deficiencies of natural anticoagulants, AT deficiency has the lowest prevalence (0.02\%-0.2\% in the general population). ${ }^{1,13}$ This deficiency is considered the most severe among the inherited thrombophilias. AT deficiency is transmitted with an autosomal dominant trait and its penetrance is very high. A wide variety of mutations can lead to type I defects, characterized by decreased activity and antigen levels. Type II defects, caused by missense mutations, are functional defects associated with normal AT antigen levels but with impaired inhibitory activity due to the production of a variant protein. ${ }^{1,6,13}$ The dysfunction may affect the reactive site $\left(\mathrm{II}_{\mathrm{RS}}\right)$ or the heparin-binding site $\left(\mathrm{II}_{\mathrm{HBS}}\right)$ or both (pleiotropic effect, $\mathrm{II}_{\mathrm{PE}}$ ). The reactive site, which is located at the carboxy-terminal part of the protein, is encoded by exon 7 of the SERPINC1 gene, while the heparin-binding site is encoded by exons 2 and $3 .{ }^{1,7}$ In the study by Wypasek et al, ${ }^{12}$ the causative mutation was found in 26 of 35 patients with AT deficiency, leading to a mutation detection rate of $74 \%$. In individuals with AT activity below $70 \%$, the mutation detection rate was $90 \%$. In $50 \%$ of the patients, mutations were located 
at exon 2 or exon 7 . These results confirm previous findings in other populations that molecular genetic testing is a useful diagnostic tool for confirming inherited AT deficiencies, although in other populations molecular testing was suggested in the AT activity range of $70 \%$ to $80 \%$ as well. ${ }^{7}$ Moreover, a genetic analysis can be particularly helpful when it comes to the differentiation between AT deficiency subtypes, thus facilitating patient care. Among the newly detected SERPINC1 gene mutations described by Wypasek et $a l,{ }^{12}$ type I deficiency is likely in the case of p.Glu338*, p.Val458_Cys462del insGly, c.948delC, p.Thr433Ser and c.625-2A>G mutations. The new p.Ala118Pro and the p.Gly125Cys variants are located in highly conserved serpin residues and thus are likely to result in the disturbance of the correct folding of AT.

PC and PS deficiencies are transmitted as autosomal dominant traits with incomplete penetrance. ${ }^{1,7}$ More than 250 loss-of-function mutations have been reported in the PC gene (PROC), located at chromosome 2q13-q14. ${ }^{6}$ Over $150 \mathrm{mu}-$ tations have been identified so far in the PROS1 gene, responsible for inherited PS deficiency. ${ }^{7}$ The majority of these reported mutations are missense mutations, short deletions or insertions, but large deletions have been identified to be relatively common as well. Both deficiencies have similar clinical presentation: heterozygotes experience early and recurrent episodes of venous thromboembolism, while the extremely rare cases of homozygotes exhibit the severe clinical picture of neonatal purpura fulminans. ${ }^{1,6,7}$ PC deficiency is classified on the basis of the plasma levels of the enzymatic activity and antigen, and, similarly to AT deficiency, it can be divided into 2 subtypes: in the case of type I deficiency, there is a parallel reduction in the PC activity and antigen levels, while in the case of the much rarer type II deficiency, normal antigen levels are accompanied by reduced functional activity. ${ }^{14}$ As for PS deficiency, 3 types have been described: type I is a quantitative deficiency with decreased plasma levels of functional and immunoreactive total and free PS, type II is a qualitative deficiency with decreased cofactor activity but normal total and free PS levels, while type III is a quantitative disorder with reduced functional activity and free PS levels but normal total PS levels. 1,6,14 It has been proposed by several studies that type I and III deficiencies are in fact phenotypic variants of the same genetic disease. ${ }^{7}$

Due to a number of methodological issues, the diagnosis of AT/PC/PS deficiencies can be a challenging task. Functional assays of all 3 proteins have several advantages and disadvantages. ${ }^{7,13,14}$ For the correct measurement of AT activity, bovine thrombin- and FXa inhibition-based tests are both advisable, as no single product appears to recognize all functional defects. ${ }^{12,13}$ Moreover, for the detection of type $\mathrm{II}_{\mathrm{HBS}}$ defects, progressive AT assays, performed in the absence of heparin, are also useful. ${ }^{13}$ PC activity can be measured by either clotting-based assays or chromogenic types of assays: in both cases, a number of interfering factors are known, leading to a potential overestimation or underestimation of PC activity. ${ }^{14}$ Measurement of PS levels is further complicated by the fact that PS is partitioned in plasma between free functional PS and the portion bound to the complement protein, $C 4 \mathrm{~b}$ -binding protein. When testing for PS deficiency, free PS antigen level is considered the "functional" anticoagulant fraction of PS (although it is not a true measure of activity) and the clottingbased PS activity assay is not recommended for the initial screening of PS levels. ${ }^{14}$ Importantly, in patients with the factor $\mathrm{V}$ Leiden mutation, PC and PS activities as measured by commercial clotting assays might be falsely decreased, leading to a potential misdiagnosis of type II PC or PS deficiency. ${ }^{7}$

Given the complexity of the diagnosis, the usefulness of molecular genetic analysis has been emphasized not only for AT deficiency but also for PC and PS deficiencies. Unfortunately, as compared with AT deficiency, the mutation detection rate by Sanger sequencing is often low for PC and PS deficiencies, suggesting that testing of patients with PC levels above $70 \%$ and free PS levels above $55 \%$ might not be expedient. ${ }^{7}$ This is in line with the findings by Wypasek et al, ${ }^{12}$ reporting that for $\mathrm{PC}$ levels below $70 \%$, the mutation detection rate was above $90 \%$, while for free PS levels below $40 \%$, the mutation detection rate was $77 \%$. It is also important to mention that the molecular diagnostics of PS deficiency is often complicated by the presence of PROS2, a pseudogene. ${ }^{1,7}$ In the study by Wypasek et al, ${ }^{12} 8$ PROC mutations and 3 PROS1 mutations were reported for the first time. The majority of the newly detected $P R O C$ gene mutations (Cys387Tyr, p.Val434Ala, and p.Leu320Pro) clustered in exon 9 , within the region encoding the catalytic domain. These variants may potentially affect substrate binding, leading to type II disorders. The newly found p.Cys64Tyr mutation, located in the gamma-carboxyglutamic domain of the PC structure and the p.Cys175Arg mutation, located in the EGF-2 domain most probably lead to type I PC deficiency. Another novel mutation, p.Gln226*, may yield a truncated protein product. Among the new mutations described in the PROS1 gene, the p.Cys126Gly and the p.Cys $241^{*}$ are located in the EGF-like 1 and the EGF-like 3 calcium-binding domains, respectively, most probably leading to type I deficiencies. Instead, in case of the newly discovered p.Gly489Arg mutation, located in the laminin-G-like 2 domain, the C4BP-binding sites are likely to be comprised, leading to type III disorder.

Today, consensus is still lacking as to who, when, and how should be tested for thrombophilia. ${ }^{8,15}$ More research is needed in order to facilitate method development and to clarify many unresolved topics regarding the rare inherited forms. If genetic analysis helps answer any of 
the questions raised, its execution is definitely timely and worthwhile.

Acknowledgments ZB is supported by the János Bólyai Fellowship of the Hungarian Academy of Sciences, OTKA PD111929 and Lajos Szodoray Prize of the University of Debrecen.

\section{REFERENCES}

1 Emmerich J, Martine MA, Morange PE. Thrombophilia Genetics. In Marder VJ, Aird WC, Benett JS, Schulman S, White GC, eds. Hemostasis and Thrombosis: Basic Principles and Clinical Practice (ed 6th). Philadelphia: Lippincott Williams and Wilkins; 2012: 962-972.

2 Egeberg 0 . Inherited antithrombin deficiency causing thrombophilia. Thromb Diath Haemorrh. 1965; 13: 516-530.

3 Sas G, Blasko G, Banhegyi D, et al. Abnormal antithrombin III (antithrom bin III "Budapest“) as a cause of a familial thrombophilia. Thromb Diath Haemorrh. 1974; 32: 105-115.

4 Griffin JH, Evatt B, Zimmerman TS, et al. Deficiency of protein C in congenital thrombotic disease. J Clin Invest. 1981; 68: 1370-1373.

5 Comp PC, Esmon CT. Recurrent venous thromboembolism in patients with a partial deficiency of protein S. N Engl J Med. 1984; 311: 1525-1528.

6 Mannucci PM, Franchini M. Classic thrombophilic gene variants Thromb Haemost. 2015; 114: 885-889.

7 Bereczky Z, Gindele R, Speker M, Kallai J. Deficiencies of the natural anticoagulants - novel clinical laboratory aspects of thrombophilia testing EJIFCC. 2016; 27: 130-146.

8 De Stefano V, Rossi E. Testing for inherited thrombophilia and consequences for antithrombotic prophylaxis in patients with venous thromboembolism and their relatives. A review of the Guidelines from Scientific Societies and Working Groups. Thromb Haemost. 2013; 110: 697-705.

9 Celinska-Lowenhoff M, Iwaniec T, Alhenc-Gelas M, et al. Arterial and venous thrombosis and prothrombotic fibrin clot phenotype in a Polish family with type 1 antithrombin deficiency (antithrombin Krakow). Thromb Haemost. 2011; 106: 379-381.

10 Wypasek E, Pankiw-Bembenek 0, Potaczek DP, et al. A missense mutation G109R in the PROC gene associated with type I protein $C$ deficiency in a young Polish man with acute myocardial infarction. Int J Cardiol. 2013 167: e146-e148.

11 Wypasek E, Alhenc-Gelas M, Undas A. First report of a large PROS1 deletion from exon 1 through 12 detected in Polish patients with deep-vein thrombosis. Thromb Res. 2013; 132: 143-144.

12 Wypasek E, Corral J, Alhenc-Gelas M, et al. Genetic characterization of antithrombin, protein $\mathrm{C}$, and protein $\mathrm{S}$ deficiencies in Polish patients. Pol Arch Intern Med. 2017; 127: 512-523.

13 Muszbek L, Bereczky Z, Kovacs B, Komaromi I. Antithrombin deficiency and its laboratory diagnosis. Clin Chem Lab Med. 2010; 48: S67-S78.

14 Marlar RA, Gausman JN. Laboratory testing issues for protein C, protein S, and antithrombin. Int J Lab Hematol. 2014; 36: 289-295.

15 Moll S. Thrombophilia: clinical-practical aspects. J Thromb Thrombolysis. 2015; 39: 367-378 